\title{
Malignant colorectal polyps: venous invasion and successful treatment by endoscopic polypectomy
}

\author{
J M Geraghty, C B Williams, I C Talbot
}

\begin{abstract}
We reviewed the pathology of 81 malignant colorectal polyps in 80 patients treated by endoscopic polypectomy and assessed the importance of carcinomatous invasion of veins in the stalk (submucosa). All the patients were followed up for at least five years. Venous invasion was present in 30 of the polyps (37\%). The histological features of lymphatic invasion were considered too subjective to be of value. Most of the tumours were well or moderately differentiated adenocarcinomas, one was poorly differentiated, and one was a signet ring cell carcinoma. Seventy one patients were treated by polypectomy alone, and 58 of these were alive and well five years later, with no evidence of recurrence. Nine died of unrelated causes within five years, but four died of carcinomatosis: one with recurrent tumour, one with a possible metachronous caecal cancer, and in two patients there was late development of malignancy of uncertain nature. The remaining nine patients underwent surgical resection after initial endoscopic polypectomy because of incompleteness of excision, poor differentiation of the tumour, or a decision by the surgeon. Tumour was not present in the resection specimens apart from a single lymph node deposit in the patient with signet ring cell carcinoma. These nine patients were alive and well without evidence of recurrence five years later. The results reemphasise the necessity of good cooperation between endoscopist and pathologist, meticulous laboratory technique, strict histopathological criteria including examination of resection margins and degree of differentiation of the tumour, and regular endoscopic follow up. Endoscopic polypectomy of pedunculated and sessile malignant polyps is adequate treatment if the lesion can be removed in one piece, the tumour is well or moderately differentiated, and local excision is judged complete by endoscopic and histological criteria. Patients with histologically incompletely excised polyps, containing well or moderately differentiated carcinoma, can be safely managed by conservative treatment provided the endoscopist is certain there is no residual tumour. Venous invasion by tumour is a common finding in malignant colorectal polyps and seems to have no prognostic importance.
\end{abstract}

Most adenocarcinomas of the colon and rectum arise in pre-existing adenomas.' In the early stages of malignancy there will clearly be an adenomatous polyp with a focus of invasive adenocarcinoma. Carcinoma is recognised in this situation when tumour has infiltrated through the muscularis mucosae into the submucosa. The lesion continues to show the morphology of a polyp - that is, a 'malignant polyp' - until a classical ulcerated invasive adenocarcinoma develops.'

The reported frequency of residual tumour or lymph node metastasis in patients with malignant colorectal polyps treated by endoscopic polypectomy lies between 0 and $15 \% .^{2-11}$ Certain histological criteria are thought to be of prognostic importance, the most widely accepted being the grade of the carcinoma and the completeness of excision. ${ }^{2+68-11}$

Several investigators have claimed that lymphatic and venous invasion are also important features, which independently determine metastatic potential in malignant polyps. ${ }^{2+581112}$ This has not been our experience at St Mark's Hospital, where endoscopic snare polypectomy is regarded as safe and effective treatment for malignant colorectal polyps provided the tumour is not poorly differentiated or incompletely excised.$^{6}$ The aim of the present study, therefore, was to assess the prognostic importance of vascular invasion in a large series of patients with malignant polyps.

\section{Methods}

For this study a malignant polyp was defined as an adenoma, part or all of which was replaced by invasive adenocarcinoma. Those polyps in which there was no residual adenoma were termed polypoid adenocarcinomas. Invasion was defined as the infiltration of adenocarcinoma through the muscularis mucosae into the stalk (submucosa).

The study material consisted of 81 malignant polyps removed from 80 patients between 1972 and 1984 at St Mark's Hospital. Seventy two of these were pedunculated and nine were sessile or broad based. The polyps were removed by snare polypectomy through an endoscope ( 73 polyps) or a rigid proctosigmoidoscope (seven polyps). A sessile malignant polyp in the rectum of one patient was excised surgically by submucosal resection (this patient also had a pedunculated rectal malignant polyp removed by snare polypectomy on the same occasion).

Seventy two of the polyps were removed in one piece, and nine in two or more pieces. They were fixed in buffered formalin and embedded and processed by methods standard at this hospital, which have been fully described in an earlier paper. ${ }^{6}$ The polyps were sectioned at multiple levels and stained with haematoxylin and eosin.

The polyps were examined histologically for each of the following features: (a) Type of adenoma: tubular, tubulo-villous or villous. 


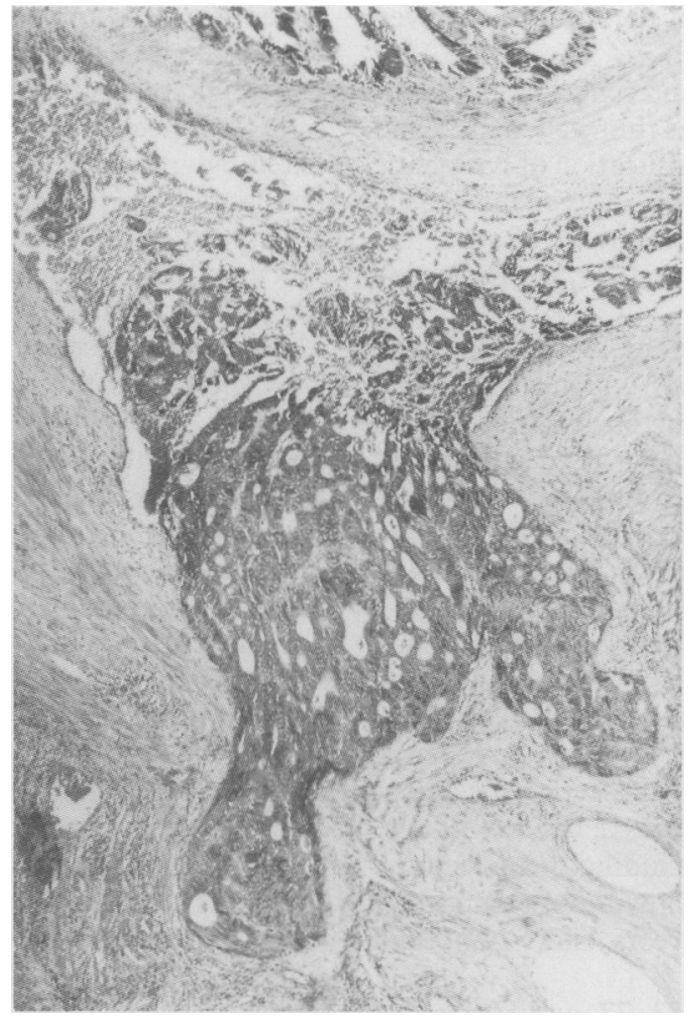

Figure 1: Adenocarcinoma invading veins in the stalk of a polyp. The top of the field includes a portion of the surface adenoma, with muscularis mucosae.

(b) Type of adenocarcinoma: adenocarcinoma without special features, mucinous carcinoma or signet ring cell carcinoma. (c) Grade of adenocarcinoma: well, moderately, or poorly differentiated. (d) Presence of venous invasion. (e) Presence of lymphatic invasion. (f) Assessment of adequacy of excision.

Venous invasion was recognised by components of the vein wall surrounding the tumour. ${ }^{13}$ The veins were designated thick or thin walled according to the amount of smooth muscle present. Thin walled veins had little smooth muscle in their walls because they were either of venular type or the vein wall was thinned due to aneurysmal dilatation by tumour (Fig 1). A recognisable endothelial lining was an essential defining feature in thin walled veins, and in any vein close to the muscularis mucosae, where tumour could be surrounded by muscle, thus simulating venous invasion. Away from this area, in the stalk, adenocarcinoma surrounded by a prominent band of smooth muscle, without recognisable endothelium, was considered sufficient evidence of thick walled venous invasion. The presence of red blood cells within channels was useful, but not diagnostic, as it might also be accounted for by areas of haemorrhage. Venous invasion was usually only seen in one or two of multiple levels, and could be recognised easily with haematoxylin and eosin staining. Elastin van Gieson staining was used in some cases but identified no further examples of venous invasion because sections deeper in the block usually did not contain the same feature of interest.

Endothelium lined channels containing tumour without luminal red blood cells or muscle in their walls were considered to be examples of lymphatic invasion. Tissue retraction around tumour could simulate the appearance of lymphatic invasion, and was considered a major potential source of error.

Completeness of excision was assessed in the light of both endoscopic and histological criteria. Those polyps removed in one piece were embedded whole in the wax block, and sectioned longitudinally. At the level of the stalk multiple sections at intervals of $0.2 \mathrm{~mm}$ were taken to minimise sampling error. Histological identification of the diathermied base then allowed assessment of the adequacy of excision. Excision of the polyp in two or more pieces could allow an opinion on completeness of excision only if the stalk was included in one of the pieces and properly orientated. In general, piecemeal polypectomy enabled only the diagnosis to be made and gave no guarantee of adequate excision.

Sessile polyps were embedded whole, or first cut into pieces if large. The entire lesion was always examined. The diathermied tissue at the margin of excision in the submucosa was used as a marker for completeness of excision.

The sections were examined histologically by one of the authors (JMG), without prior knowledge of the clinical picture or follow up data. Any examples of vascular invasion were assessed by a second pathologist (ICT) and only documented as positive if there was full agreement.

\section{Results}

The study included 80 patients. There were 34 men, mean age $67 \cdot 1$ years (range $26-81$ years), and 46 women, mean age 60.4 years (range $24-80$ years).

Eighty one malignant polyps were studied (one patient had two malignant polyps removed from the rectum during the same procedure). The distribution of polyps throughout the colon and rectum is shown in Table $\mathrm{I}$. The mean maximum diameter for all the polyps was $16.9 \mathrm{~mm}$ (range 7-35 mm) (Table I). The largest pedunculated and sessile polyps removed in one piece were 35 and $15 \mathrm{~mm}$ maximum diameter respectively. The diameters of the nine sessile polyps ranged from 8 to $25 \mathrm{~mm}$.

The histological typing of the polyps is given in Table II. Pre-existing adenomas were found in 60 of the polyps. The remaining 21 were polypoid adenocarcinomas. The carcinomas comprised 74 adenocarcinomas without special features, six mucinous carcinomas, and one signet ring cell carcinoma; 79 tumours were well or moderately differentiated. Only two polyps contained high grade tumours; the signet ring cell carcinoma and a poorly differentiated adeno-

TABLE II Histological typing and grade of polyps No $(\%)$

\section{Histology:}

Cancer in tubular adenoma Cancer in tubulovillous adenoma

Cancer in villous adenoma

Grade:

Low/well differentiated

$12(14 \cdot 8)$
$21(25 \cdot 9)$

Average/moderately differentiated

$40(49 \cdot 4)$

High/poorly differentiated (including signet ring cell carcinoma) 
TABLE III Vascular invasion

Vascular invasion $\quad \mathrm{No}(\%)$

Thin walled venous $27(33 \cdot 3)$ Thick walled venous $3(3 \cdot 7)$ Lymphatic carcinoma. The grading of the tumours is given in Table II.

Venous invasion was present in 30 polyps (37\%) (Table III). This was thin walled in 27 and thick walled in three. Identification of lymphatic invasion was a subjective assessment, easily confused with tissue retraction around the tumour, and is documented for completeness rather than accuracy.

Figure 2 shows completeness of excision and venous invasion in relation to management and prognosis. Sixty three polyps from 62 of the patients were judged to have been completely excised by histological and endoscopic criteria (these include the two polyps excised from the rectum of the same patient). Venous invasion was present in 21 of the polyps (from 21 patients), including three with tumour in thick walled veins. One patient had several additional adenomas distributed throughout the colon and immediately underwent a total colectomy. There was no residual tumour in the resection specimen. The remainder were managed conservatively. Fifty of these patients, including the colectomy patient, were alive and well without evidence of recurrence five or more years later. Nine had died of unrelated causes.

Three elderly patients $(73,77$, and 80 years at the time of polypectomy) who had had well or moderately differentiated malignant polyps removed endoscopically, died of carcinomatosis five years, four years, and 18 months after polypectomy. Venous invasion was observed in only one of these. The eldest patient, who had had a malignant polyp of the sigmoid colon, refused any follow up examination after initial limited colonoscopy (to the splenic flexure) and polypectomy, but terminally had a mass in the caecum; and others had normal follow up colonoscopy one and 29 months later, respectively. None had a postmortem examination.

The polyps in 18 patients were considered to

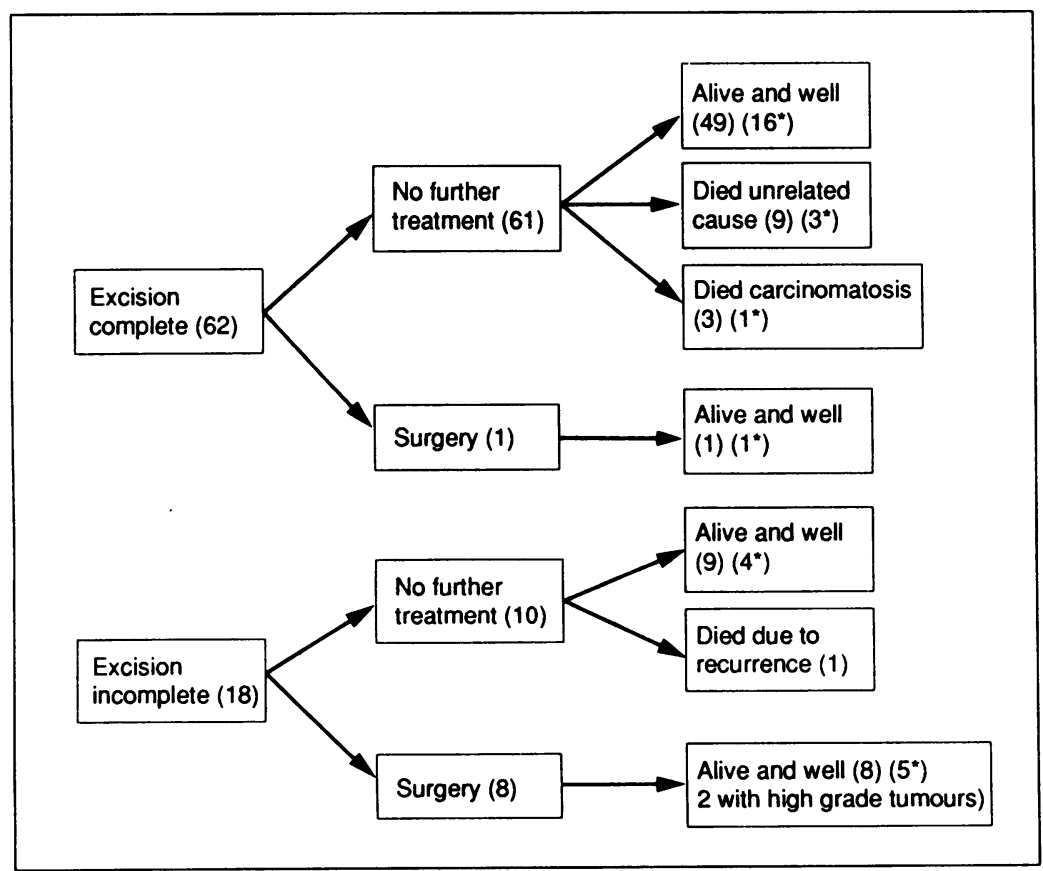

Figure 2: Flow chart of histopathology, venous invasion, and prognosis in 80 patients. ${ }^{\star}$ Venous invasion found. have been incompletely excised; thin walled venous invasion was present in nine of these. Only eight patients (including the patients with signet ring cell carcinoma and poorly differentiated adenocarcinoma) underwent a surgical resection. Residual tumour was not present in seven of the resection specimens. The resection specimen from the patient with signet ring cell carcinoma contained a small deposit of tumour in a lymph node close to the polypectomy ulcer, but was otherwise free of tumour. The remaining 10 patients were followed up endoscopically. Seventeen of these patients were alive and well at least five years later. One patient with a $25 \mathrm{~mm}$ sessile polyp, incompletely excised by piecemeal polypectomy and showing well differentiated mucinous carcinoma on histology, was initially not submitted to surgery when three week reexamination with multiple biopsies showed no residual tumour at the polypectomy site. At one year follow up, however, there was definite evidence of recurrence and surgery was carried out. The resection specimen showed mucinous adenocarcinoma, similar to the original tumour, spreading through the wall into the sub-serosa without lymph node involvement (Dukes's stage B). The patient died seven months later of widespread metastatic disease.

There were nine sessile polyps in the series. Five of these, measuring $15 \mathrm{~mm}$ or less, were removed in one piece, each containing well or moderately differentiated adenocarcinoma. In all five the excision margins were clear of invasive tumour. All five patients were alive without recurrence at least five years later. Larger sessile polyps from four patients, measuring 20 to $25 \mathrm{~mm}$ diameter, were removed by piecemeal polypectomy and excision was histologically uncertain in each case. Immediate surgical resection was carried out in two of these patients, but the other two were managed conservatively. Three patients remained well without recurrence at five years. The fourth died of recurrent mucinous carcinoma and is described above.

\section{Discussion}

The follow up data are similar to those reported by others, ${ }^{246810}$ and confirm that excellent results are possible if a carefully controlled policy of polypectomy for malignant polyps is pursued. Surgical resection need only be considered if the tumour is of high grade or excision is incomplete. There has effectively been only one dissenting report, ${ }^{3}$ with a $15 \%$ frequency of residual tumour among 39 patients. The latter study, however, included surgical resection material and advanced carcinomas, and the principal author, therefore, subsequently admitted that it was not comparable to the other studies of endoscopic polypectomy. 9

The rationale behind this policy is based on the low frequency, about $10 \%$, of lymph node metastasis in patients with adenocarcinomas confined to the submucosa at the time of surgical resection. ${ }^{14}$ Most of the tumours which do metastasise are poorly differentiated. High grade tumours are particularly uncommon in malignant polyps, ${ }^{611}$ numbering two in this series, and should be treated by surgical resection if this is 
clinically feasable. The patient in the present study with signet ring cell carcinoma, a high grade tumour associated with a poor prognosis, ${ }^{15}$ had a single lymph node deposit in the resection specimen. This patient is alive and well without evidence of recurrence over 15 years later. It is notable that studies of similar colorectal carcinomas (Dukes's stage $C$ tumours in which the growth is confined to the bowel wall) have shown a prognosis comparable to stage B carcinomas. ${ }^{1617}$

The single most important criterion for predicting residual tumour is the adequacy of excision. ${ }^{6}$ This can be assessed histologically by identification of the diathermy burn, which represents the plane of excision. Correct orientation of the polyp within the wax block is essential, and sections at multiple levels should be examined. For this to be reliably achieved the polyp should be removed as a total excisional biopsy in one piece. This limits the number of lesions amenable to polypectomy; the maximum diameters of pedunculated and sessile polyps snared in one piece in this study were $35 \mathrm{~mm}$ and $15 \mathrm{~mm}$ respectively. Conservative management of small (under $15 \mathrm{~mm}$ ) sessile polyps, like pedunculated lesions, seems therefore to be entirely adequate, in keeping with the findings of others. ${ }^{10}$

The four sessile polyps measuring between 20 and $25 \mathrm{~mm}$ and five pedunculated lesions between 10 and $30 \mathrm{~mm}$ were resected piecemeal, one of the patients in this group dying later of recurrent tumour. Piecemeal polypectomy entails increased risk, being difficult to assess endoscopically and difficult to orientate for sectioning. Histology can give no guarantee of adequate excision in this situation.

The results indicate that continued conservative management of patients with histologically incompletely excised polyps, containing well or moderately differentiated adenocarcinoma, is safe provided the endoscopist is certain that no residual tumour is present. Only one of the 10 patients managed in this way developed recurrent disease. A further six patients, who came to surgery with incompletely excised well or moderately differentiated adenocarcinomas, had no residual tumour in the resection specimens, and, in retrospect, could also have been treated conservatively. These findings may be related to destruction of tumour at the plane of excision by the diathermy. Conservative management of patients with incompletely excised polyps containing well or moderately differentiated adenocarcinomas clearly entails increased risk but may be justified in the treatment of elderly patients or those with other contraindications to surgery. The mortality immediately after 'curative' surgical resection for colorectal carcinoma is $3 \%$ and $12 \%$ for patients under and over 70 years, respectively. ${ }^{18} \mathrm{Had}$ all the elderly patients in this series undergone surgical resection, therefore, it is likely that as many or more would have died postoperatively, probably without carcinoma being present.

Only one patient definitely died of recurrent tumour in this series. The polyp was sessile, measuring $25 \mathrm{~mm}$, and removed piecemeal so that excision could not be guaranteed. Surgery was delayed because no residual tumour was detected endoscopically or histologically at three week follow up. The recurrence in this patient may also relate to the histology of the tumour, a mucinous carcinoma, which has been shown by others to lead a more aggressive course than ordinary adenocarcinoma. ${ }^{15}$ This may merit further studies. Five other patients, however, with completely excised mucinous carcinomas were successfully treated by polypectomy alone.

Regarding the three elderly patients who died with a diagnosis of 'carcinomatosis', recurrent malignancy from the original polyp cannot be ruled out as necropsies were not performed. The subsequent outcome may, however, be related to non-colorectal primaries or synchronous or metachronous tumours. Elderly patients with colorectal index lesions are at risk for either of these possibilities. ${ }^{19}$ The polyps contained well and moderately differentiated adenocarcinomas and were completely excised. Two of the patients lived four and five years after polypectomy and had negative follow up colonoscopy. The third patient, who had had a limited initial examination (to the splenic flexure) and refused follow up, was noted to have a mass in the caecum, suggesting that this was the likely primary.

Venous invasion in the stalk or submucosa was a common finding in malignant polyps in this study. This high frequency has not been reported previously and may relate to the careful search in multiple sections carried out in each case. Venous invasion was present in $52 \%$ of 703 rectal adenocarcinomas examined at St Mark's Hospital, ${ }^{13}$ most commonly in submucosal (intramural) veins; $33 \%$ of Dukes's B and C tumours showed venous invasion outside the muscle coats (extramural). Intramural venous invasion had no effect on five year survival figures for Dukes's A, $B$, and $C$ cases, but the prognosis was appreciably worsened by extramural venous invasion, particularly if thick walled veins were involved. As would be expected, since the invading margin in malignant polyps is confined to the submucosa, venous invasion, whether in thin or thick walled veins, conferred no increased risk of recurrence in patients in the present series.

Our findings conflict with those of Muller et $a l,{ }^{12}$ the only other published study specifically dealing with venous and lymphatic invasion. The series comprised 34 patients with malignant polyps, six (17.6\%) of whom had demonstrable vascular invasion (five venous). Ten were submitted to surgery and residual or metastatic tumour was present in all the resection specimens, four of the original polyps showing vascular invasion. A fifth patient with vascular invasion who did not have surgery developed recurrent tumour. Two of the patients with vascular invasion would have been submitted to surgery anyway because their polyps were considered to be incompletely excised. The remaining three patients, however, had completely excised polyps containing moderately differentiated adenocarcinoma and would normally have been managed conservatively. Statistical analysis showed vascular invasion to be an adverse prognostic factor. There are several problems with this study which make us question its validity. The study group was small and lacked uniformity 
as the cases were obtained from three different hospitals. The sizes of the polyps with vascular invasion and whether or not these were removed piecemeal were not specified. A rate of recurrence of $32 \%$ is one of the highest published and conflicts with the experience at St Mark's and other institutions.

Several investigators have claimed that lymphatic invasion is an adverse prognostic indicator in malignant polyps. ${ }^{2+5811}$ Only few malignant polyps with this feature, however, have been reported, often with confounding factors of incomplete excision or high grade tumour. We have found that assessment of lymphatic invasion was too subjective to draw any valid conclusion.

We confirm the findings of others that endoscopic snare polypectomy is safe and effective treatment for patients with malignant polyps, provided certain criteria are strictly applied. The polyps should be snared in one piece to ensure complete excision as judged endoscopically and histologically, emphasising the need for meticulous laboratory technique and good cooperation between pathologist and endoscopist. In general, conservative management should, therefore, be confined to pedunculated and sessile polyps with maximum diameters of about 35 and $15 \mathrm{~mm}$ respectively. Patients with contraindications to surgery can be safely managed by conservative treatment even when excision seems histologically to be incomplete, provided the adenocarcinoma is well or moderately differentiated and the endoscopist is certain there is no residual tumour. Patients with high grade tumours should undergo surgical resection. Mucinous carcinomas may confer increased risk of recurrence, but further studies need to be carried out to confirm this. The presence of venous invasion has no adverse effect on prognosis.
1 Morson BC, Dawson IMP, Day DW, Jass JR, Price AB, Williams GT. Morson and Dawson's gastrointestinal pathology London: Blackwell, 1990: 574-6.

2 Wolff WI, Shinya H. Definitive treatment of malignant polyps of the colon. Ann Surg 1975; 182: 516-25.

3 Colacchio TA, Forde KA, Scantlebury VP. Endoscopic polypectomy - inadequate treatment for invasive colorectal carcinoma. Ann Surg 1981; 194: 704-7.

4 Rossini FP, Ferrari A, Spandre M, Coverlizza S. Colonoscopic polypectomy in diagnosis and management of cancerous adenomas: an individual and multicentre experience. Endoscopy 1982; 14: 124-7.

5 Cooper HS. Surgical pathology of endoscopically removed malignant polyps of the colon and rectum. Am $\mathcal{F}$ Surg Pathol 1983; 7: 613-23.

6 Morson BC, Whiteway JE, Jones EA, MacRae FA, Williams CB. Histopathology and prognosis of malignant colorectal polyps treated by endoscopic polypectomy. Gut 1984; 25: polyps trea $437-44$.

7 Hagitt RC, Glotzbach RE, Soffer EE, Wruble LD. Prognostic factors in colorectal carcinomas arising in adenomas: implicafactors in colorectal carcinomas arising in adenomas: implica-
tions for lesions removed by endoscopic polypectomy. tions for lesions removed by endo
Gastroenterology 1985; 89: 328-36.

8 Cranley JP, Petras RE, Carey WD, Paradis K, Sivak MV. When is endoscopic polypectomy adequate therapy for colonic polyps containing invasive carcinoma? Gastroenterology 1986; 91: 419-27.

9 Wilcox GM, Anderson PB, Colacchio TA. Early invasive carcinoma in colonic polyps. A review of the literature with emphasis on the assessment of the risk of metastasis. Cancer 1986; 57: 160-71.

10 Christie JP. Polypectomy or colectomy? Management of 106 consecutively encountered colorectal polyps. Am Surgeon consecutively enc

11 Coverlizza S, Risio M, Ferrari A, Fenoglio-Preiser CM, Rossini FP. Colorectal adenomas containing invasive car cinoma. Pathologic assessment of lymph node metastatic potential. Cancer 1989; 64: 1937-47.

12 Muller S, Chesner IM, Egan MJ, et al. Significance of venous and lymphatic invasion in malignant polyps of the colon and rectum. Gut 1989; 30: 1385-91.

13 Talbot IC, Ritchie S, Leighton M, Hughes AO, Bussey HJR, Morson BC. Invasion of veins by carcinoma of rectum: method of detection, histological features and significance. Histopathology 1981; 5: 141-63.

14 Morson BC. Factors influencing the prognosis of early cancer of the rectum. Proceedings of the Royal Society of Medicine of the rectum. Pro

15 Sasaki O, Atkin WS, Jass JR. Mucinous carcinoma of the rectum. Histopathology 1987; 11: 259-72.

16 Phillips RKS, Hittinger R, Blesovsky L, Fry JS, Fielding LP. Large bowel cancer. Surgical pathology and its relationship to survival. BrF Surg 1984; 71: 604-10.

17 Jass JR, Love SB. Prognostic value of direct spread in Dukes C cases of rectal cancer. Dis Colon Rectum 1989; 32: 477-80

18 Fielding LP, Phillips RKS, Hittinger R. Factors influencin mortality after curative resection for large bowel cancer in elderly patients. Lancet 1989; i: 595-7.

19 Grossman S, Milos ML, Tekawa IS, Jewell DP. Colonoscopic screening of persons with suspected risk factors for colon cancer: II. Past history of colorectal neoplasms. Gastroenterology 1989; 96: 299-306. 